\title{
Caracterización clínica y microbiológica de las infecciones causadas por Streptococcus grupo anginosus
}

\author{
GUSTAVO CARO D., INGRID RIEDEL K. y PATRICIA GARCÍA C.
}

\section{Clinical and microbiological characteristics of infections caused by Streptococcus anginosus group}

Streptococcus anginosus group and its species constellatus, anginosus and intermedius, produce suppurative infections. Its microbiological identification is complex because it can be $\beta$-hemolytic and shares Lancefield antigens with other Streptococci. This group grows as small colonies and presents a characteristic smell, but if only latex test for carbohydrate $\mathrm{C}$ agglutination is used it can be erroneously classified. The aims of this publication were to evaluate an algorithm that includes three biochemical tests, and to describe in vitro susceptibility to antibiotics and clinical characteristics of infections caused by Streptococcus anginosus group. Streptoccoccal strains belonging to groups C, G and $\mathrm{F}$ were evaluated, determining its colony characteristics in order to select small colony strains that were included into the proposed low cost algorithm (beta-glucurosidase and Voges Proskauer tests) comparing them with API Rapid ID 32 Strept ${ }^{\circledR}$ method, considered the gold standard. Two hundred nineteen strains were studied, 101 had a morphological profile compatible with the anginosus group, identifying S. constellatus in 58\% (59/101), S. anginosus in 37\% (37/101) and S. intermedius in 5\% (5/ 101). Strains were isolated mainly from pyogenic infections $(61 \%, 62 / 101)$, specially from abdominal origin. High grade of penicillin and cefotaxime susceptibility (98\%) and low grade resistance to erithromycin and clindamycin (6,8 and 5\% respectively) were observed. Mean patient age was 30 years old (2-85), a similar sex distribution with predominance of inpatients. These results document the usefulness of the proposed low cost algorithm for detection of $S$. anginosus group. In our experience this bacterial group maintains good susceptibility to penicillin and cefotaxime, and we recommend a continous surveillance of clindamycin and erythromycin susceptibility.

Key words: Streptococcus anginosus; Classification; Infections; In vitro susceptibility.

Palabras claves: Streptococcus grupo anginosus; Clasificación; Infecciones; Susceptibilidad in vitro.

\section{Introducción}

Streptococcus grupo anginosus corresponde a un grupo de cocáceas grampositivas, dispuestas en cadena, cuya clasificación, terminología e identificación microbiológica ha resultado compleja a través del tiempo.

Forma parte de la flora normal de la cavidad oral, tracto gastrointestinal y genitourinario y se asocia con el desarrollo de infecciones purulentas ${ }^{1}$.
Dentro de este grupo se describen tres distintas especies: S. anginosus, S. constellatus y S. intermedius $^{2,4}$ y en la literatura médica se asocia a ciertas localizaciones más frecuentes: $S$. intermedius en infecciones del SNC e hígado, S. constellatus en infecciones pulmonares u otras localizaciones y $S$. anginosus en sangre, tracto gastrointestinal e infecciones de tejidos blandos ${ }^{5-6}$. Estas caracterizaciones no han estado exentas de discrepancia, así como el rol patógeno de este grupo en distin-

Pontificia Universidad Católica de Chile.

Escuela de Medicina (GCD).

Laboratorio de Microbiología. Unidad Docente Asociada de Laboratorios Clínicos (IRK, PGC).

Recibido: 3 junio 2004

Aceptado: 29 junio 2004 
tos cuadros clínicos. Un ejemplo de lo anteriormente expuesto se relaciona a su participación en el desarrollo de faringitis ${ }^{7-9}$; ha sido necesario la subclasificación de especie para clarificar este punto, identificando finalmente a Streptococcus constellatus subsp pharyngis subs nov con un rol etiológico real en esta patología ${ }^{10}$.

Para lograr la identificación de este grupo se ha utilizado clásicamente sus características de hemólisis ( $\alpha, \beta$ o no hemolítica), la determinación de los antígenos de Lancefield (A, C, G y F) y las características macroscópicas de la colonia: pequeña y con olor característico a caramelo ${ }^{1,2}$; el uso como criterio único de antígenos de Lancefield en la clasificación de muestras faríngeas puede llevar a errores al identificar Streptococcus grupo "milleri” como Streptococcus pyogenes $^{11}$. Para la identificación definitiva de especie, Whiley y colaboradores ${ }^{12}$ han propuesto la utilización de siete pruebas bioquímicas que también permiten la identificación de subespecies. Otras alternativas son los sistemas comerciales con sustratos liofilizados (API ${ }^{\circledR}$, bioMerieux) que tienen buena correlación con el método de Whiley pero, por su alto costo, son inaccesibles en países como el nuestro ${ }^{3}$. Kirby y colaboradores propusieron en 1995 una alternativa de bajo costo para la identificación de Streptococcus grupo anginosus que, si bien no permite el diagnóstico de especie, permite determinar el grupo en muestras faríngeas ${ }^{13}$. Con independencia de la hemólisis, la importancia de la correcta clasificación de este grupo radica en la asociación con infecciones purulentas que pueden requerir resolución quirúrgica (abscesos) y que las pruebas de susceptibilidad a los antimicrobianos, según las recomendaciones de la NCCLS, son homologables a las del grupo viridans ${ }^{14}$. La secuenciación del gen que codifica para $16 \mathrm{~S}$ rARN ha permitido establecer que $S$. intermedius y $S$. constellatus están genotípicamente muy relacionados y que no siempre existe correlación entre la identificación fenotípica (bioquímica) y la genoespecie ${ }^{4}$.

La susceptibilidad a penicilina de este grupo no ha presentando grandes variaciones en los distintos estudios publicados ${ }^{15,16}$, si bien Bantar y colaboradores han reportado $13 \%$ de resistencia a penicilina en S. anginosus y 5\% en S. constellatus ${ }^{17}$. La susceptibilidad a macrólidos, cefalosporinas y clindamicina ha sido variable ${ }^{18}$ y las sulfonamidas son inactivas ${ }^{15}$.

Dado lo anteriormente descrito los objetivos de este trabajo fueron evaluar un algoritmo de bajo costo para la identificación de Streptococcus grupo anginosus, describir la susceptibilidad a los antimicrobianos y caracterizar a los pacientes con infecciones causadas por estos microorganismos.

\section{Material y Métodos}

Se realizó una revisión a partir de la base de datos del Laboratorio de Microbiología de la Red de Salud de la Pontificia Universidad Católica de Chile desde agosto de 1999 a febrero del 2004. Se incluyeron cepas de Streptococcus sp $\beta$ hemolíticas de los grupos C, F y G de Lancefield y cepas no $\beta$-hemolíticas informadas como Streptococcus grupo anginosus. Se excluyeron del análisis las especies formadores de colonia grande: $S$. pyogenes (pues a todas las cepas $\beta$ hemolíticas se les realizó el test de pirrolidonil aminopeptidasa (PYR) y sólo se incluyeron las PYR negativas) y Streptococcus agalactiae (única especie que aglutina como grupo B). Las cepas permanecían congeladas a $-80^{\circ} \mathrm{C}$ en caldo glicerol con suero de caballo y se descongelaron, realizando un doble traspaso en agar sangre de cordero al $5 \%$ e incubándose a $35^{\circ} \mathrm{C}$ durante 16 horas.

Se registraron las características morfológicas de las colonias (definiendo como colonia grande aquella mayor de $0,5 \mathrm{~mm}$ de diámetro y como colonia pequeña aquella menor de $0,5 \mathrm{~mm}$ ), la hemólisis presentada en agar sangre de cordero al 5\% y la presencia o ausencia de olor característico (semejante a caramelo).

A todas las cepas se les realizó test de PYR, test de látex para Streptococcus $\beta$-hemolíticos (Streptex ${ }^{\circledR}$, Murex) según las recomendaciones del fabricante, test de Voges-Proskauer (VP) y $\beta$-glucuronidasa (BGUR) según Kirby y colaboradores $^{13}$. Siguiendo el esquema de este autor, a las cepas BGUR negativo y VP positivo, se les realizó API Rapid ID 32 S Strept ${ }^{\circledR}$ (bioMerieux, Haz, Mo)

Test de Voges-Proskauer. Permite detectar la presencia de acetil-metil-carbinol, producido por Streptococcus grupo anginosus. Para realizar el procedimiento se toma con tórula abundante cantidad de cepa (Mc Farland 5) a partir de una siembra en agar sangre de cordero al 5\%, incubada previamente durante 16 horas. Se inocula en caldo rojo de metilo o caldo VP, se incuba durante 6 horas a $35^{\circ} \mathrm{C}$ y se le agrega $0,6 \mathrm{ml}$ de solución de $\mathrm{KOH}$ al $40 \%$ (40 g de hidróxido de potasio disuelto en $100 \mathrm{ml}$ de agua destilada) y $0,2 \mathrm{ml}$ de solución de $\alpha$ naftol al 5\% (5 g de $\alpha$ naftol disuelto en $100 \mathrm{ml}$ de etanol), agitándose después de cada adición. Se considera reacción positiva la formación de un producto de color rojo-rosado, 
a los 30 minutos de la incubación a temperatura ambiente. Se considera negativo si no ocurre el cambio de color antes de este tiempo.

Test de $\boldsymbol{\beta}$-glucuronidasa. Mide la reacción de BGUR, enzima producida por Streptococcus grupo $\mathrm{C}$ y $\mathrm{G}$ colonias grandes pero no por el grupo anginosus. A partir de una siembra en agar sangre de cordero al 5\% incubada previamente durante 16 horas, se prepara una emulsión concentrada (Mc Farland 4) en $250 \mu \mathrm{L}$ de cloruro de sodio al 9\%o. Se agrega a esta emulsión una tableta de BGUR (Tabletas Diatabs ${ }^{\circledR}$, Rosco Diagnóstica), se agita hasta la disolución de la tableta y se incuba durante 18 horas a $35-37^{\circ} \mathrm{C}$. Se considera positiva la presencia de color amarillo.

API Rapid ID 32 Strept ${ }^{\circledR}$ (bioMerieux, Haz, Mo). Sistema estandarizado semiautomático, que incluye 32 ensayos enzimáticos miniaturizados con reactivos deshidratados. Para preparar el inóculo se toman colonias del cultivo en agar sangre preparando una suspensión ajustada al diodo $\mathrm{N}^{\circ} 30$ del densitómetro ATB ${ }^{\circledR}$ o 4 de McFarland en el Densimat ${ }^{\circledR}$. Luego se incuba a $36 \pm 2^{\circ} \mathrm{C}$ durante 4 a 4 1/2 horas en aerobiosis. Después de la incubación, las reacciones se llevan al lector, cuyos resultados se interpretan en una base de datos específica.

Los estudios de susceptibilidad para el grupo anginosus se realizaron según las recomendaciones del NCCLS $2004^{14}$, utilizando E-test para evaluar CIM en penicilina y cefotaxima y Kirby Bauer (difusión en disco) para eritromicina (discos de $15 \mu \mathrm{g}$ ) y clindamicina. Se consideró sensible (S), intermedio (I) y resistente (R) de acuerdo a las guías NCCLS como sigue: penicili-

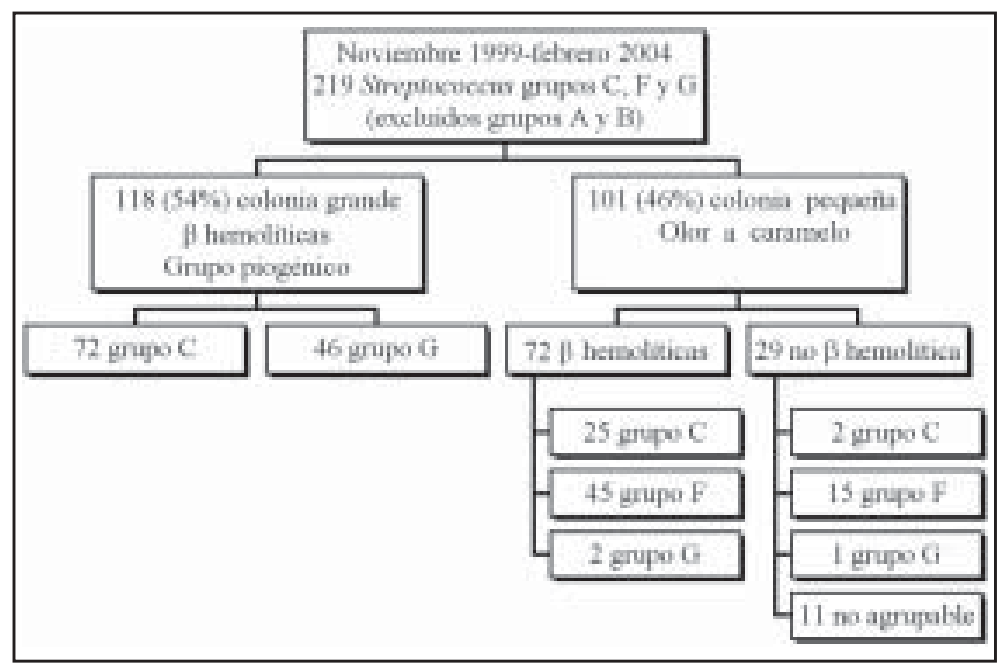

Figura 1. Distribución de Streptococcus grupo C, F y G según tamaño de la colonia, hemólisis y agrupación por antígenos de Lancefield. na $(\mu \mathrm{g} / \mathrm{ml}), \mathrm{S}<0,12$ I entre 0,25 y 2 y $\mathrm{R}>4$ y cefotaxima $(\mu \mathrm{g} / \mathrm{ml}) \mathrm{S}<1$, I 2 y $\mathrm{R}>4$. Para eritromicina (mm) $\mathrm{S}>21$, I entre $16-20$ y $R<15$ y para clindamicina $(\mathrm{mm}) \mathrm{S}>19$, I entre $16-18$ y $\mathrm{R}<15$.

En los pacientes en quienes se identificó Streptococcus grupo anginosus se obtuvo información de la edad, sexo, procedencia (ambulatorio/hospitalizado), tipo de muestra, localización de la infección y presencia de infección polimicrobiana. Se revisó la ficha clínica de los pacientes internados con infecciones clínicamente significativas, registrándose además los días de hospitalización y la duración de la terapia antimicrobiana.

\section{Resultados}

Se incluyeron en el estudio 219 cepas de Streptococcus grupo C, F y G, 118 (54\%) correspondieron a colonias grandes, $\beta$-hemolíticas (72 grupo C y 46 grupo G) y 101 (46\%) correspondieron a colonias pequeñas, siendo $72 \beta$-hemolíticas ( 25 grupo $C, 45$ grupo $F$ y 2 grupo $G$ ) y 29 no $\beta$ hemolíticas (2 grupo $\mathrm{C}, 15$ grupo $\mathrm{F}, 1$ grupo $\mathrm{G} y$ 11 no agrupable) (Figura 1 ).

En las 101 cepas de Streptococcus colonia pequeña con test PYR negativo, el test de BGUR fue negativo y el VP fue positivo, concordante con la identificación por API Rapid ID 32 Strept ${ }^{\circledR}$. La especie más frecuentemente identificada fue S. constellatus en 58\% (59/101), en segundo lugar S. anginosus en 37\% (37/101) y por último, $S$. intermedius en 5\% (5/101) (Figura 2). La relación entre las especies identificadas y los grupos de Lancefield se observa en la Tabla 1, donde destaca que 69,5\% (41/ 59) de las cepas identificadas como S. constellatus, aglutinaron con el grupo F, correspondiendo 16 a muestras peritoneales, 11 a muestras faríngeas, 9 a abscesos y 5 a otras localizaciones.

Los resultados de los estudios de susceptibilidad se muestran en la Tabla 2, donde se observa que $98 \%$ de los Streptococcus grupo anginosus son susceptibles a penicilina (99/ 101) y cefotaxima $(99 / 101)$, en cambio existe $6,9 \%$ de cepas resistentes a eritromicina (7/ 101) y $5 \%$ de cepas resistentes 


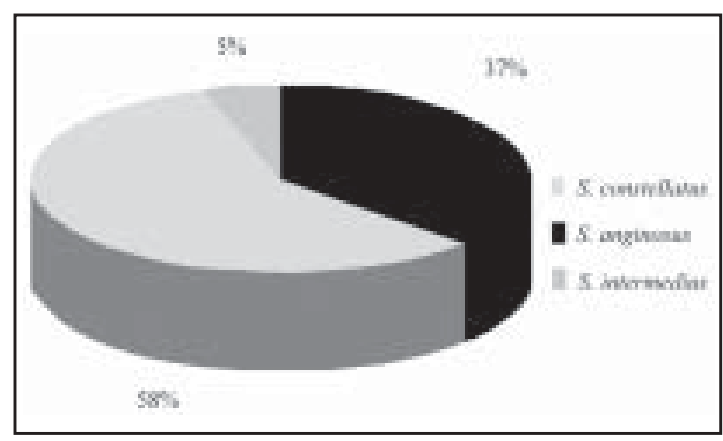

Figura 2. Distribución de especies en las 101 cepas de Streptococcus grupo anginosus, según identificación por API Rapid ID Strep®.

a clindamicina (5/101). No hubo en la serie cepas resistentes a penicilina, sólo 2 con resistencia intermedia.

Las 101 muestras provenían de pacientes cuya edad promedio era 30 años (edades extremas 2 y 85 años), con distribución similar por sexo (52 hombres y 49 mujeres) y con predominio de pacientes hospitalizados (66/101). De los 101 pacientes, 47 cursaron con infección por Streptococcus grupo anginosus como agente único y 54 cursaron con una infección polimicrobiana; de éstas, 37 presentaron un solo microorganismo además de Streptococcus grupo anginosus y 17 presentaron dos o más. Con mayor frecuencia acompañaron al grupo anginosus, Escherichia coli, Pseudomonas aeruginosa, Klebsiella pneumonia y Haemophilus spp; si se consideran los anaerobios en conjunto, éstos ocupan el segundo lugar (Tabla 3). La distribución según localización de la infección por especie bacteriana se describe en la Tabla 4, en la que se observa que la infección más frecuente por grupo anginosus fue la abdominal (41/101), seguida de las infecciones de cabeza y cuello (32/101). Destaca que 62\% (63/101) de las infecciones del grupo anginosus correspondían a infecciones piogénicas de cavidades estériles o abscesos. En la distribución por especie, tanto $S$. constellatus como $S$. anginosus se asociaron más frecuentemente a infecciones abdominales. Es destacable también que $23 \%$ de las muestras correspondían a frotis faríngeo (23/101).

De los 66 pacientes hospitalizados, se obtuvo información a partir de la ficha clínica en 49, siendo el promedio de días de hospitalización de $10,8 \pm 8,8$ días y el promedio de días de tratamiento antimicrobiano $16,7 \pm 8,4$ días (dato obtenido en 39 pacientes).

\section{Discusión}

La aplicación de un algoritmo que utiliza el tamaño de la colonia, el olor característico, la hemólisis y los tests PYR, BGUR y VP es un excelente método de bajo costo para la identifica-

Tabla 1. Relación entre las especies identificadas y los grupos de Lancefield

\begin{tabular}{lcccrr}
\hline $\begin{array}{c}\text { Grupo anginosus } \\
\text { según especie }\end{array}$ & Grupo C & Grupo de Lancefield & Grupo G & No agrupable & Total \\
\hline S. constellatus & $12^{*}$ & $41^{* *}$ & 1 & 5 & 59 \\
S. anginosus & 12 & 19 & 2 & 4 & 37 \\
S. intermedius & 3 & - & - & 2 & 5 \\
Total & 27 & 60 & 3 & 11 & 101 \\
\hline
\end{tabular}

* Correspondían a muestras de abscesos (5), faríngeas (4) y otras localizaciones (3).

** Correspondían a muestras peritoneales (16), faríngeas (11), abscesos (9) y otras localizaciones (5).

Tabla 2. Susceptibilidad antimicrobiana en las 101 cepas estudiadas de Streptococcus grupo anginosus

\begin{tabular}{lcccc}
\hline Antimicrobianos & Sensibles & $\begin{array}{c}\text { Susceptibilidad in vitro } \\
\text { Intermedios }\end{array}$ & Resistentes & Total \\
\hline Penicilina* $^{*}$ & 99 & 2 & - & 101 \\
Cefotaxima* & 99 & 1 & 1 & 101 \\
Eritromicina** & 93 & 1 & 7 & 101 \\
Clindamicina** & 96 & - & 5 & 101 \\
\hline
\end{tabular}

* Método de E-test (concentración inhibitoria mínima).

** Método de Kirby Bauer (difusión en disco). 
Tabla 3. Caracterización de la población estudiada e información microbiológica $(n=101)$

\begin{tabular}{|c|c|c|}
\hline \multicolumn{3}{|c|}{ Características descriptivas } \\
\hline Edad (años) & \multicolumn{2}{|c|}{$30(2-85)$} \\
\hline $\operatorname{Sexo}(\mathrm{H}: \mathrm{M})$ & \multicolumn{2}{|c|}{ 52: 49} \\
\hline \multirow[t]{2}{*}{ Procedencia } & Ambulatorios & 35 \\
\hline & Hospitalizados & 66 \\
\hline \multirow[t]{4}{*}{ Tipo de muestra } & Sangre & 3 \\
\hline & Abscesos & 62 \\
\hline & Secreciones & 13 \\
\hline & Faringe & 23 \\
\hline \multirow[t]{16}{*}{ Tipo de infección } & Monomicrobiana & 47 \\
\hline & Polimicrobiana & 54 \\
\hline & E. coli & 30 \\
\hline & P. aeruginosa & 7 \\
\hline & Haemophilus sp & 6 \\
\hline & Otras Enterobacterias & 6 \\
\hline & Prevotella $\mathrm{sp}$ & 5 \\
\hline & S. aureus & 4 \\
\hline & Peptostreptococcus & 4 \\
\hline & K. pneumoniae & 3 \\
\hline & Otros Streptococcus & 3 \\
\hline & S. coagulasa (-) & 3 \\
\hline & Enterococcus sp & 1 \\
\hline & A. lwoffi & 1 \\
\hline & C. albicans & 1 \\
\hline & Lactobacillus sp & 1 \\
\hline
\end{tabular}

ción de Streptococcus grupo anginosus. Esto permite una aproximación diagnóstica de grupo con gran utilidad para laboratorios que no disponen de sistemas comerciales y que puede evitar el uso del test de látex para Streptococcus en cepas macroscópicamente sospechosas ${ }^{19}$. Este algoritmo fue descrito por Kirby y colaboradores ${ }^{13}$, sólo para cepas $\beta$-hemolíticas, recomendándose para cepas no $\beta$-hemolíticas agregar el test de $\operatorname{arginina}^{1}$. Nuestros resultados en las 29 cepas no $\beta$-hemolíticas, fueron, sin embargo, concordantes con la identificación definitiva.

Al igual como lo describe la literatura médica, las especies más frecuentemente aisladas a partir de muestras clínicas fueron $S$. constellatus y $S$. anginosus, siendo menos frecuente el aislamiento de $S$. intermedius ${ }^{5}$; sin embargo, dado que se ha descrito que el sistema API Rapid ID32 StreptÒ puede identificar sólo $57 \%$ las cepas de $S$. intermedius, las cifras de aislamiento de esta especie podrían estar subestimadas ${ }^{3}$. Respecto de la asociación entre especie y grupo de Lancefield nuestros resultados son coincidentes con la literatura médica sobre la preponderancia del grupo $\mathrm{F}^{20} \mathrm{y}$ también con Facklam y colaboradores $^{3}$, quienes describen que $S$. constellatus se asocia especialmente con los grupos $\mathrm{C}$ y $\mathrm{F}$ (en nuestra serie correspondieron a 20 y $69,5 \%$ de $S$. constellatus, respectivamente). Según estos autores, las cepas que aglutinan con grupo $\mathrm{C}$, corresponden a la subespecie pharyngis. En nuestros resultados, para la especie constellatus, 33\% (4/12) de las cepas que poseían antígeno $\mathrm{C}$, provenían

Tabla 4. Distribución de las especies de Streptococcus grupo anginosus según localización de la infección

\begin{tabular}{|c|c|c|c|c|}
\hline \multirow{2}{*}{$\begin{array}{l}\text { Localización de } \\
\text { la infección }\end{array}$} & \multicolumn{3}{|c|}{ Especie de Streptococcus grupo anginosus } & \multirow[t]{2}{*}{ Total } \\
\hline & S. constellatus & S. anginosus & S. intermedius & \\
\hline Pleuropulmonar & 2 & 1 & - & 3 \\
\hline $\begin{array}{l}\text { Cabeza y cuello } \\
\text { Faringe } \\
\text { Abscesos }\end{array}$ & $\begin{array}{r}15 \\
4\end{array}$ & $\begin{array}{l}7 \\
2\end{array}$ & $\begin{array}{l}1 \\
3\end{array}$ & $\begin{array}{r}23 \\
9\end{array}$ \\
\hline $\begin{array}{l}\text { Abdominal } \\
\text { Peritonitis } \\
\text { Abscesos }\end{array}$ & $\begin{array}{r}23 \\
3\end{array}$ & $\begin{array}{r}13 \\
2\end{array}$ & $\begin{array}{l}- \\
-\end{array}$ & $\begin{array}{r}36 \\
5\end{array}$ \\
\hline Piel y tejidos blandos & 2 & 7 & - & 9 \\
\hline $\begin{array}{l}\text { Ginecológicos } \\
\text { Abscesos } \\
\text { Secreción vaginal }\end{array}$ & $\begin{array}{l}3 \\
2\end{array}$ & $\begin{array}{l}1 \\
-\end{array}$ & $\overline{1}$ & $\begin{array}{l}4 \\
3\end{array}$ \\
\hline Sangre & 1 & 2 & - & 3 \\
\hline Abscesos & 4 & 2 & - & 6 \\
\hline Total & 59 & 37 & 5 & 101 \\
\hline
\end{tabular}




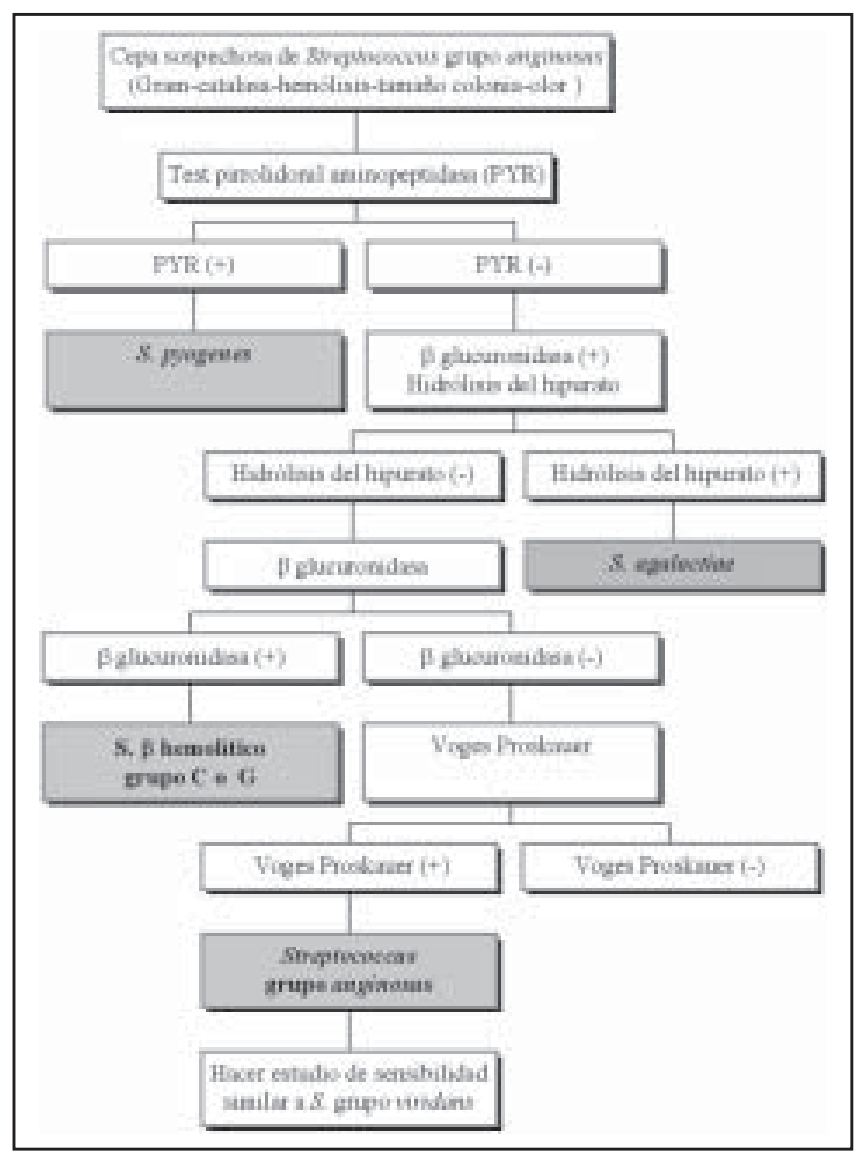

Figura 3. Algoritmo de bajo costo para la identificación de Streptococcus grupo anginosus.

de muestras faríngeas (Tabla 1). Según Facklam, 30 a 55\% de Streptococcus grupo anginosus aglutinan para antígeno $\mathrm{F}^{3}$, coincidente con $59 \%$ (60/101) del grupo F encontrado en el presente trabajo; en cambio, la mayoría (61\%) de Streptococcus del grupo piogénico, colonia grande, corresponden al grupo C (72/118) (Figura 1).

La susceptibilidad antimicrobiana encontrada es semejante a la referida previamente por la literatura médica, destacando una excelente susceptibilidad a penicilina y cefotaxima y una mayor resistencia a eritromicina y clindamicina. Esto demuestra que es necesaria la vigilancia periódica de resistencia a macrólidos y clindamicina (grupo MLS: macrólidos, lincosamidas, streptograminas). Creemos que es necesario conocer los mecanismos por los cuales este grupo bacteriano es resistente al grupo MLS en nuestro medio, así como se ha descrito para S. pyogenes ${ }^{22}$.

La caracterización clínica de los pacientes permite establecer igual a lo descrito previamen$t^{22}$, el rol principalmente piogénico de este grupo de microorganismos, lo que explica por qué $65 \%$ de los pacientes requirió una hospitalización para la resolución de la infección, con una estadía promedio prolongada y terapia antimicrobiana durante al menos 10 días. No encontramos una relación entre el sitio de afectado por la infección y la especie patógena especifica del grupo ya que tanto para $S$. constellatus como para S. anginosus la localización más frecuente de la infección fue la abdominal, especialmente asociada a peritonitis. En la localización faríngea no se pudo establecer el rol patogénico de $S$. constellatus por la falta de acceso a información clínica, todos estos pacientes procedían de la comunidad y por otro lado, se sabe que $S$. constellatus es parte de la flora faríngea habitual. Es necesario realizar un estudio prospectivo de casos y controles para aclarar el rol patogénico de $S$. constellatus como agente causal de faringitis.

En nuestra serie destaca, tal como se describe en la literatura médica, la importante asociación de Streptococcus grupo anginosus con otros patógenos bacterianos (polimicrobismo) junto a anaerobios y E.coli, explicado por la alta frecuencia en nuestra serie de infecciones abdominales.

Por último, nos parece importante destacar que desde el punto de vista clínico este grupo de Streptococcus debe ser considerado como un agente etiológico probable en infecciones piogénicas, siendo $47 \%$ de las infecciones descritas en este estudio monomicrobianas y 3 casos bacteriémicas.

Si bien el grupo se ha mantenido susceptible a los antimicrobianos, es necesario continuar con la vigilancia de la resistencia y por ultimo, nos parece de gran importancia la validación de una estrategia de laboratorio de bajo costo y alta eficacia para la identificación de Streptococcus grupo anginosus (Figura 3).

\section{Resumen}

Streptococcus grupo anginosus, y sus especies constellatus, anginosus e intermedius, causan infecciones supuradas. Su identificación microbiológica es compleja pues pueden ser $\beta$-hemolíticos y comparten antígenos de Lancefield con otros estreptococos. Si bien morfológicamente son colonias pequeñas y presentan un olor característicos, si sólo se realiza test de látex para aglutinación del carbohidrato $\mathrm{C}$ pueden ser clasificados erróneamente. El objetivo de nuestro trabajo fue evaluar un 
algoritmo que utiliza 3 pruebas bioquímicas, conocer su susceptibilidad a antimicrobianos y describir las características clínicas de infecciones producidas por Streptococcus grupo anginosus. Se estudiaron cepas de Streptococcus grupo C, G y F, determinando sus características morfológicas de colonia, para identificar cepas de colonia pequeña que se incluyeron en el algoritmo de bajo costo propuesto ( $\beta$ glucuronidasa y el test Voges-Proskauer) comparándose al método API Rapid ID 32 Strept ${ }^{\circledR}$, establecido como estándar de oro. Se estudiaron 219 cepas, de las cuales 101 presentaron las características morfológicas compatibles con grupo anginosus, identificándose $S$. constellatus en $58 \%$ (59/101), S. anginosus en $37 \%(37 / 101)$ y S. intermedius en $5 \%$ (5/101). Las cepas aisladas procedían principalmente de infecciones piogénicas $(61 \%, 62 / 101)$, en especial de foco abdominal. Se observó $98 \%$ de susceptibilidad a penicilina y cefotaxima y resistencia a eritromicina y clindamicina en 6,8 y $5 \%$, respectivamente. La edad promedio de los pacientes fue de 30 años (2-85), con distribución similar por sexo y predominio de pacientes hospitalizados.

Los resultados expuestos demuestra la efectividad del algoritmo de bajo costo para la detección de S. grupo anginosus. En nuestra experiencia este grupo bacteriano conserva su susceptibilidad a penicilina y cefotaxima, siendo necesario vigilar clindamicina y eritromicina.

\section{Bibliografía}

1.- Ruoff K L, Whiley R A, Brighton D. Streptococcus, In: P.R. Murray, E.J. Baron, J.H. Jorgensen, M.A. Pfaller, R.H. Yolken (ed). Manual of Clinical Microbiology, $8^{\text {th }}$ ed. American Society of Microbiology, Washington DC. 2003. p. 405-21.

2.- Whiley R A, Beighton D, Winstanley T G, Fraser H Y, Hardie J M. Streptococcus intermedius, Streptococcus constellatus, and Streptococcus anginosus (the Streptococcus milleri group): association with different body sites and clinical infections. J Clin Microbiol 1992; 30: 243-4.

3.- Facklam R. What happened to the Streptococci: Overview of taxonomic and nomenclature changes. Clin Microbiol Rev 2002; 15: 613-30.

4.- Clarridge J E, Osting C, Jalali M, Osborne J, Waddington M. Genotypic and phenotypic characterization of Streptococcus milleri group isolates from a Veterans Administration hospital population. J Clin Microbiol 1999; 37: 3681-7.

5.- Clarridge J E, Attorri S, Musher D M, Hebert J, Dunbar S. Streptococcus intermedius, Streptococcus constellatus, and Streptococcus anginosus ("Streptococcus milleri Group): Are of different clinical importance and are not equally associated with the abscess. Clin Infect Dis 2001; 32: 1511-5.

6.- Belko J, Goldmann D A, Macone A, Zaidi A K. Clinically significant infections with organisms of the Streptococcus milleri group. Pediatr Infect Dis J 2002; 21: 715-23.

7.- Fox J, Turner, A Fox. Role of Beta-hemolytic group C Streptococci in pharyngitis: Incidence and biochemical characteristics of Streptococcus equisimilis and Streptococcus anginosus in patients and healthy controls. J Clin Microbiol 1993; 31: 804-7.
8.- Turner J C, Fox A, Fox K, Addy C, Garrison C Z, Herron B, Brunson C, Betcher G. Role of group C beta-hemolytic streptococci in pharyngitis: epidemiologic study of clinical features associated with isolation of group C streptococci. J Clin Microbiol 1993; 3: 808-11.

9.- Turner J C, Hayden F G, Lobo M C, Ramírez C E, Murren D. Epidemiologic evidence for Lancefield group $\mathrm{C}$ beta-hemolytic streptococci as a cause of exudative pharyngitis in college students. J Clin Microbiol 1997; 35: 1-4.

10.- Whiley, Hall, Hardie J M, Beighton D. A study of small-colony, beta-haemolytic, Lancefield group C streptococci within the anginosus group: description of Streptococcus constellatus subsp. pharyngis subsp. nov, associated with the human throat and pharyngitis. Int J Syst Bacteriol 1999; 49: 1443-9.

11.- Hamrick H J, Mangum M E. Beta-hemolytic Streptococcus milleri group misidentified as Streptococcus pyogenes on throat culture. Pediatr Infect Dis J 1999; 18: 75-6.

12.- Whiley R A, Fraser H, Hardie J M, Beighton D. Phenotypic differentiation of Streptococcus intermedius, Streptococcus constellatus, and Streptococcus anginosus strains within the "Streptococcus milleri group". J Clin Microbiol 1990; 28: 1497-501.

13.- Kirby R, Ruoff K L. Cost-effective, clinically relevant method for rapid identification of beta-hemolytic streptococci and enterococci. J Clin Microbiol 1995; 33: 1154-7.

14.- National Committee for Clinical Laboratory Standards. 2004. Performance standard for antimicrobial susceptibility testing; Fourteenth informational supplement. M100-S14, January 2004.

15.- Tracy M, Wanahita A, Shuhatovich Y, Goldsmith E A, Clarridge J E 3rd, Musher D M. Antibiotic susceptibilities of genetically characterized Streptococcus milleri group strains. Antimicrob Agents Chemother 2001; 1511-4.

16.- Aracil B, Gómez-Garcés J L, Alos J I. A study of susceptibility of 100 clinical isolates belonging to the Streptococcus milleri group to 16 cephalosporins. J Antimicrob Chemother 1999; 43: 399-402.

17.- Bantar C, Fernández L, Relloso S, Lanza A, Bianchini $\mathrm{H}$, Smayevsky. Species belonging to the "Streptococcus milleri" group: antimicrobial susceptibility and comparative prevalence in significant clinical specimens. J Clin Microbiol 1996; 34: 2020-2.

18.- Jacobs J A, van Baar G J, London N H, Tjhie J H, Schouls L M, Stobberingh E E. Prevalence of macrolide resistance genes in clinical isolates of the Streptococcus anginosus ("S. milleri") group. Antimicrob Agents Chemother 2001; 45: 2375-7.

19.- Flynn C E, Ruoff K L. Identification of "Streptococcus milleri" group isolates to the species level with a commercially available rapid test system. J Clin Microbiol 1995; 33: 2704-6.

20.- Whitworth J M. Lancefield group F and related streptococci. J Med Microbiol 1990; 33: 135-51.

21.- Palavecino E L, Riedel I, Berrios X, Bajaksouzian S, Johnson D, Kaplan E, Jacobs M R. Prevalence and mechanisms of macrolide resistance in Streptococcus pyogenes in Santiago, Chile. Antimicrob Agents Chemother 2001; 45: 339-41.

22.- Ruoff K L. Streptococcus anginosus ("Streptococcus milleri"): the unrecognized pathogen. Clin Microbiol Rev 1988; 1: 102.

Correspondencia a:

Patricia García Cañete

E-mail: pgarcia@med.puc.cl 